\title{
Comunicación
}

\section{Valores Hematológicos y Bioquímica Sérica del Caimán Blanco (Caiman crocodilus) de Vida Libre en Madre de Dios, Perú}

\author{
Hematologic and Serum Biochemistry Values in Free-Living Spectacled Caiman \\ (Caiman crocodilus) in MAdre de Dios, Peru
}

Nancy Carlos ${ }^{13}$, Yoana Nuñez del Prado², Daniela Quispe ${ }^{3}$, Carmen Capuñay ${ }^{2}$

\section{Resumen}

\begin{abstract}
El objetivo del estudio fue determinar los valores hematológicos y bioquímica sérica del caimán blanco (Caiman crocodilus) de vida libre en Madre de Dios, Perú. Se capturaron 30 individuos (26 machos y 4 hembras) en las márgenes del río Madre de Dios. Las muestras sanguíneas se obtuvieron por punción en la rama dorsal de la vena craneal. Los valores hallados fueron: eritrocitos $1.45 \pm 0.97 \times 10^{6} / \mu 1$, hematocrito $22.63 \pm 4.16 \%$, hemoglobina $7.86 \pm 2.10 \mathrm{~g} / \mathrm{dl}, \mathrm{VCM} 190.6 \pm 76.9 \mathrm{fl}, \mathrm{HCM} 63.49 \pm 20.96 \mathrm{pg}, \mathrm{CMCH} 34.96 \pm 7.80 \mathrm{~g} / \mathrm{dl}$, leucocitos $13.18 \pm 5.85 \times 10^{3} / \mu 1$, eosinófilos $1.54 \pm 1.21 \times 10^{3} / \mu 1$, basófilos $1.31 \pm 1.20 \times 10^{3} / \mu 1$, linfocitos $5.77 \pm 4.03 \times 10^{3} / \mu 1$, monocitos $0.17 \pm 0.38 \times 10^{3} / \mu$ l, heterófilos $2.16 \pm 2.59 \times 10^{3} / \mu 1$, ALT $48.59 \pm 14.34 \mathrm{U} / \mathrm{L}$, AST $114.0 \pm 28.5 \mathrm{UI} / \mathrm{L}, \mathrm{GGT} 1.8 \pm 1.6 \mathrm{UI} / \mathrm{L}$, creatinina $0.51 \pm 0.35 \mathrm{UI} / \mathrm{L}$, colesterol 101.8 $\pm 42.3 \mathrm{UI} / \mathrm{L}$, glucosa $98.1 \pm 30.7 \mathrm{mg} / \mathrm{dL}$, proteínas totales $5.01 \pm 1.00 \mathrm{~g} / \mathrm{dl}$, albúmina $1.65 \pm 0.29 \mathrm{~g} / \mathrm{dL}$, globulinas $3.13 \pm 1.08 \mathrm{~g} / \mathrm{dL}$, calcio $10.11 \pm 1.63 \mathrm{mg} / \mathrm{dL}$ y fósforo $6.44 \pm 1.09 \mathrm{mg} / \mathrm{dl}$. Se encontraron diferencias significativas según el sexo para los valores de VCM y CMCH.
\end{abstract}

Palabras clave: bioquímica, caimán, hematología, reptil

\section{Abstract}

The aim of the study was to determine the values of hematologic and serum biochemistry in free-living spectacled caiman (Caiman crocodilus) in Madre de Dios, Peru. A total of 30 individuals (26 males and 4 females) were captured in the banks of the

\footnotetext{
${ }^{1}$ Programa de Ecología de Enfermedades y Medicina de la Conservación, Centro de Ornitología y Biodiversidad, Lima, Perú

${ }^{2}$ Centro de Rescate Taricaya, Madre de Dios, Perú

${ }^{3}$ Facultad de Medicina Veterinaria, Universidad Alas Peruanas, Lima, Perú

${ }^{4}$ E-mail: nancy.carlos.erazo@gmail.com
}

Recibido: 10 de junio de 2016

Aceptado para publicación: 12 de octubre de 2016 
Madre de Dios river. Blood sampled were collected from the dorsal branch of the cranial vein. The following results were found: RBC $1.45 \pm 0.97 \times 10^{6} / \mu 1, \mathrm{PCV} 22.63 \pm 4.16 \%, \mathrm{Hb}$ $7.86 \pm 2.10 \mathrm{~g} / \mathrm{dl}, \mathrm{MCV} 190.6 \pm 76.9 \mathrm{fl}, \mathrm{MCH} 63.49 \pm 20.96 \mathrm{pg}, \mathrm{MCHC} 34.96 \pm 7.80 \mathrm{~g} / \mathrm{dl}, \mathrm{WBC}$ $13.18 \pm 5.85 \times 10^{3} / \mu 1$, eosinophils $1.54 \pm 1.21 \times 10^{3} / \mu 1$, basophils $1.31 \pm 1.20 \times 10^{3} / \mu 1$, lymphocytes $5.77 \pm 4.03 \times 10^{3} / \mu 1$, monocytes $0.17 \pm 0.38 \times 10^{3} / \mu 1$, heterophiles $2.16 \pm 2.59 \times 10^{3} / \mu 1$, ALT $48.59 \pm 14.34 \mathrm{U} / \mathrm{L}, \mathrm{AST} 114.0 \pm 28.5 \mathrm{UI} / \mathrm{L}, \mathrm{GGT} 1.8 \pm 1.6 \mathrm{UI} / \mathrm{L}$, creatinine $0.51 \pm 0.35 \mathrm{UI} / \mathrm{L}$, cholesterol 101.8 $\pm 42.3 \mathrm{UI} / \mathrm{L}$, glucose $98.1 \pm 30.7 \mathrm{mg} / \mathrm{dL}$ total protein $5.01 \pm 1.00 \mathrm{~g} / \mathrm{dl}$, albumin $1.65 \pm 0.29 \mathrm{~g} / \mathrm{dL}$, globulins $3.13 \pm 1.08 \mathrm{~g} / \mathrm{dL}$, calcium $10.11 \pm 1.63 \mathrm{mg} / \mathrm{dL}$, phosphorus $6.44 \pm 1.09$ $\mathrm{mg} / \mathrm{ml}$. Significant differences were found by sex for MVC and MCHC.

Key words: biochemistry, caiman, hematology, reptile

\section{INTRODUCCIÓN}

El caimán blanco (Caiman crocodilus) se distribuye mayormente en países de América Central y del Sur, incluyendo la región de Madre de Dios en Perú. Los caimanes contribuyen con la conservación del equilibrio energético, el estado sanitario del microhábitat y en la conservación de las fuentes de agua (Rueda et al., 2007).

Existen pocos estudios sobre los aspectos biológicos y sanitarios de dicho reptil. En un estudio llevado a cabo en Venezuela, se reportaron 17 valores hematológicos y bioquímicos (Rossini et al., 2011), en tanto que el Sistema Internacional de Información de Especies (International Species Information System - ISIS) reporta diversos valores en individuos en crianza en cautiverio de diversos zoológicos (ISIS, 2004).

Los estudios sobre los parámetros fisiológicos y la salud de animales silvestres han cobrado mayor importancia, debido a su utilidad, como especies indicadoras para determinar la condición y el grado de contaminación de los ecosistemas. Uno de los análisis menos invasivos para determinar el estado fisiológico y la salud de poblaciones de animales silvestres es el análisis de parámetros fisiológicos, incluyendo los valores hematológicos y de bioquímica sérica (Nagy y Medica, 1986; Martínez et al., 2013).
Debido a la importancia de los caimanes y a la falta de información biológica y biomédica, el objetivo del estudio fue determinar los valores hematológicos y de bioquímica sérica del caimán blanco (Caiman crocodilus). Estos valores servirán de ayuda para el diagnóstico de enfermedades en animales en cautiverio, para mejorar el conocimiento de la salud de esta especie, el ecosistema que habita y contribuir con la conservación de la fauna silvestre de la Amazonia peruana.

\section{Materiales y Métodos}

El estudio se llevó a cabo en el río Madre de Dios, $23 \mathrm{~km}$ al noroeste de la ciudad de Puerto Maldonado, en el departamento de Madre de Dios, Perú. El periodo de captura del caimán blanco se realizó entre noviembre y diciembre de 2014. Se capturaron 30 individuos (26 machos y 4 hembras), correspondiendo a 2 adultos, 27 juveniles y 1 subadulto. Los individuos fueron clasificados según su longitud total: cría (Clase I: $<50 \mathrm{~cm}$ ), juvenil (Clase II: 50 a $120 \mathrm{~cm}$ ), subadulto (Clase III: $>120$ a $180 \mathrm{~cm}$ ) y adulto (Clase IV: $>180 \mathrm{~cm}$ ) (Escobedo y Gonzales, 2008; Velasco y Ayarzagüena, 1995).

Se contó con el permiso para la captura y la colección de muestras biológicas, otorgado por el Servicio Forestal y de Fauna Sil- 
vestre (Resolución General N. ${ }^{\circ}$ 042-2014SERFOR-DGGDPFFS). La captura se hizo mediante contención física, y se llevó a cabo durante la noche, con botes recorriendo ambos márgenes del río y sus quebradas. Se realizó un breve examen clínico y se tomaron medidas biométricas (largo del cuerpo y largo de cola) con la ayuda de una cinta métrica. El peso fue determinado con una balanza digital de mano (WeiHeng), con una precisión de $10 \mathrm{~g}$. Se recolectó de 2 a $5 \mathrm{ml}$ de sangre de la rama dorsal de la vena cava craneal, utilizando una jeringa de $5 \mathrm{ml}$ con aguja de $21 \mathrm{G} \times 1 \frac{11 / 2}{2}$. La muestra fue dividida en frascos al vacío sin coagulante y con coagulante heparina-litio.

Las muestras fueron analizadas en un laboratorio clínico particular, especializado en muestras de animales silvestres, ubicado en la ciudad de Lima. Las muestras se trabajaron en un lapso de 36 a 38 horas después de tomadas. La concentración de hemoglobina fue determinada por espectrofotometría mediante el método de cianometahemoglobina y el hematocrito se determinó utilizando tubos de microhematocrito. El conteo de eritrocitos y leucocitos se determinó utilizando el hemocitómetro Neubauer, la solución Natt-Herrick y frotis sanguíneo teñido con Giemsa. Los once valores bioquímicos fueron determinados utilizando el analizar automático Stat Fax 1904 Plus $11 \circledR$ (Awareness Technology, EEUU). El valor de la glucosa fue determinado en campo utilizando el contador automatizado Accu Check ${ }^{\circledR}$ (Roche).

El análisis de los datos se realizó utilizando estadística descriptiva. La normalidad de los datos se determinó utilizando la prueba de Shapiro-Wilk. Para determinar las posibles diferencias según el sexo, se empleó la prueba de T-Student con un nivel de confianza del $95 \%(\mathrm{p}<0.05)$. Se utilizó el programa estadístico SPSS v. 212012 (SPSS Inc., Chicago, Illinois, USA).

\section{Resultados}

Los resultados de los valores hematológicos y bioquímicos obtenidos se muestran en los cuadros 1 y 2 , respectivamente, en tanto que los resultados hematológicos según sexo se presentan en el Cuadro 3. Los valores que fueron significativamente diferentes entre sexos correspondieron a los valores de VCM $(\mathrm{p}=0.029)$ y $\mathrm{CMHC}(\mathrm{p}=0.027)$.

\section{Discusión}

Algunos de los valores hematológicos y de bioquímica sérica hallados en este estudio fueron diferentes de los reportados para la misma especie en Venezuela (Rossini et al., 2011), diferencias que pudieron deberse mayormente a factores fisiológicos, como la época del año y la dieta (Arcila et al., 2005; Martínez et al., 2013). Los valores de GGT, creatinina, colesterol, glucosa, calcio y fosforo son nuevos reportes para esta especie en vida libre.

Rossini et al. (2011) reportaron valores menores de eritrocitos (machos $0.5 \times 10^{6} / \mu 1 \mathrm{y}$ hembras $0.6 \times 10^{6} / \mu 1$ ), posiblemente debido a las épocas y los estados fisiológicos de los animales. En tortugas se ha observado un aumento de eritrocitos tras el periodo de hibernación en época de invierno; sin embargo, en caimanes neotropicales no se ha descrito hibernación, sino solo un cierto grado de letargia durante la época húmeda (IUCN, 1986). Por otro lado, los valores de hematocrito y hemoglobina fueron bastante similares a los hallazgos en el estudio venezolano (Rossini et al., 2011). No obstante, se reporta una variación del hematocrito según la edad, sexo y efecto climático en reptiles (Arcila et al., 2005; Martínez et al., 2013). 
Cuadro 1. Valores hematológicos (promedio \pm D.E., mínimo y máximo) del caimán blanco (C. crocodilus) de vida libre en el departamento de Madre de Dios, Perú (2014)

\begin{tabular}{|c|c|c|c|c|}
\hline Valores & Promedio & D.E. & Mínimo & Máximo \\
\hline Eritrocitos $\left(10^{6} / \mu 1\right)$ & 1.45 & 0.97 & 0.55 & 5.64 \\
\hline Hematocrito (\%) & 22.63 & 4.16 & 12 & 33 \\
\hline Hemoglobina (g/dl) & 7.86 & 2.10 & 4.7 & 13.7 \\
\hline $\operatorname{VCM}^{1}(\mathrm{fl})$ & 190.6 & 76.9 & 47.8 & 392.9 \\
\hline $\mathrm{HCM}^{2}(\mathrm{pg})$ & 63.49 & 20.96 & 17.88 & 121.43 \\
\hline $\mathrm{CMCH}^{3}(\mathrm{~g} / \mathrm{dl})$ & 34.96 & 7.80 & 22.17 & 59.52 \\
\hline Leucocitos $\left(10^{3} / \mu \mathrm{l}\right)$ & 13.18 & 5.85 & 4.4 & 25.96 \\
\hline Eosinófilos $\left(10^{3} / \mu 1\right)$ & 1.54 & 1.21 & 0 & 4.74 \\
\hline Basófilos $\left(10^{3} / \mu \mathrm{l}\right)$ & 1.31 & 1.20 & 0 & 5.2 \\
\hline Linfocitos $\left(10^{3} / \mu 1\right)$ & 5.77 & 4.03 & 0.53 & 18.69 \\
\hline Monocitos $\left(10^{3} / \mu \mathrm{l}\right)$ & 0.17 & 0.38 & 0 & 1.74 \\
\hline Heterófilos $\left(10^{3} / \mu 1\right)$ & 2.16 & 2.59 & 0.4 & 11.64 \\
\hline
\end{tabular}

Cuadro 2. Valores de bioquímica sérica (promedio \pm D.E., mínimo y máximo) del caimán blanco (C. crocodilus) de vida libre en el departamento de Madre de Dios, Perú (2014)

\begin{tabular}{lcccc}
\hline Valores & Promedio & D.E. & Mínimo & Máximo \\
\hline $\operatorname{ALT}^{1}(\mathrm{U} / \mathrm{L})$ & 48.59 & 14.34 & 24 & 91 \\
$\operatorname{AST}^{2}(\mathrm{U} / \mathrm{L})$ & 114.0 & 28.5 & 72 & 202 \\
$\mathrm{GGT}^{1} 3(\mathrm{U} / \mathrm{L})$ & 1.8 & 1.6 & 0 & 6.7 \\
Creatinina $\mathrm{UI} / \mathrm{L})$ & 0.51 & 0.35 & 0.2 & 2.3 \\
Colesterol $(\mathrm{UI} / \mathrm{L})$ & 101.8 & 42.3 & 55 & 218 \\
Glucosa $(\mathrm{mg} / \mathrm{dl})$ & 98.1 & 30.7 & 48 & 161 \\
Proteínas totales $(\mathrm{g} / \mathrm{dl})$ & 5.01 & 1.00 & 2.7 & 7.1 \\
Albúmina $(\mathrm{g} / \mathrm{dl})$ & 1.65 & 0.29 & 1.1 & 2.4 \\
Globulina $(\mathrm{g} / \mathrm{dl})$ & 3.13 & 1.08 & 1.2 & 4.7 \\
Calcio $(\mathrm{mg} / \mathrm{dl})$ & 10.11 & 1.63 & 7.2 & 13.4 \\
Fósforo $(\mathrm{mg} / \mathrm{dl})$ & 6.44 & 1.09 & 5 & 9.2 \\
\hline${ }^{1}$ Alaninoamino transferasa ${ }^{2}{ }^{2}$ Aspartatoamino transferasa ${ }^{3}$ Gamma glutamil transpeptidasa
\end{tabular}


Cuadro 3. Valores hematológicos (promedio \pm D.E., mínimo y máximo) del caimán blanco (C. crocodilus) macho y hembra de vida libre en el departamento de Madre de Dios, Perú (2014)

\begin{tabular}{|c|c|c|c|c|c|c|}
\hline \multirow{2}{*}{ Valores } & \multicolumn{3}{|c|}{ Hembra $(n=4)$} & \multicolumn{3}{|c|}{ Macho $(n=26)$} \\
\hline & Promedio & D.E. & Mín-Máx & Promedio & D.E. & Mín-Máx \\
\hline Eritrocitos $\left(10^{6} / \mu 1\right)$ & 1.46 & 1.76 & $0.9-3.5$ & 1.2 & 0.97 & $0.6-5.7$ \\
\hline Hematocrito (\%) & 19.6 & 5.47 & $12.0-27.0$ & 23.24 & 3.7 & $15.0-33.0$ \\
\hline Hemoglobina (g/dl) & 7.74 & 1.89 & $4.7-9.5$ & 7.88 & 2.18 & $5.1-13.7$ \\
\hline $\operatorname{VCM}^{1}(F 1)$ & $129.28^{\mathrm{a}}$ & 51.83 & $54.3-200$ & $202.81^{\mathrm{a}}$ & 75.91 & $47.8-393$ \\
\hline $\mathrm{HCM}^{2}(\mathrm{pg})$ & 50.85 & 18.01 & $21.4-70.4$ & 66.02 & 20.9 & $17.9-121.4$ \\
\hline $\mathrm{CMCH}^{3}(\mathrm{~g} / \mathrm{dl})$ & $39.47^{\mathrm{b}}$ & 3.2 & $35.2-43.9$ & $32.73^{\mathrm{b}}$ & 8.12 & $27.9-59.5$ \\
\hline Leucocitos $\left(10^{3} / \mu 1\right)$ & 16.08 & 10.41 & $4.8-26.0$ & 12.6 & 4.96 & $4.4-21.8$ \\
\hline Eosinófilos $\left(10^{3} / \mu 1\right)$ & 0.71 & 0.45 & $0.3-3.1$ & 1.34 & 1.24 & $0-4.7$ \\
\hline Basófilos $\left(10^{3} / \mu \mathrm{l}\right)$ & 0.71 & 1.13 & $0.3-3.1$ & 0.93 & 1.23 & $0-3.5$ \\
\hline Linfocitos $\left(10^{3} / \mu \mathrm{l}\right)$ & 7.36 & 6.8 & $1.1-18.7$ & 4.69 & 3.2 & $0.5-12.9$ \\
\hline Monocitos $\left(10^{3} / \mu \mathrm{l}\right)$ & 0.14 & 0.32 & $0-0.7$ & 0.17 & 0.4 & $0-1.7$ \\
\hline Heterófilos $\left(10^{3} / \mu 1\right)$ & 2.60 & 3.72 & $0-11.7$ & 2.06 & 2.34 & $0-7.0$ \\
\hline
\end{tabular}

${ }^{1}$ Volumen corpuscular medio; ${ }^{2}$ Hemoglobina Corpuscular Media; ${ }^{3}$ Concentración de hemoglobina corpuscular media

${ }^{a, b}$ Superíndices diferentes indican diferencia estadística $(p<0.05)$ entre promedios por sexo

Los valores de VCM y CMHC fueron mayores que los valores descritos por Rossini et al. (2011); sin embargo, se describe un amplio rango de VCM (160-950 fl) en reptiles (Martínez et al., 2013). El valor de $\mathrm{CMCH}$ disminuye en casos de anemia por deficiencia de hierro, dieta o pérdida de sangre (Copete-Sierra, 2013).

Rossini et al. (2011) observaron menores valores de leucocitos $\left(10.3 \times 10^{3} / \mu 1\right)$, monocitos $\left(0.82 \times 10^{3} / \mu 1\right)$ y heterófilos $\left(5.4 \times 10^{3} /\right.$ $\mu 1)$, pero mayores valores de basófilos $\left(0.5 \times 0^{3} /\right.$ $\mu 1)$ y linfocitos $\left(2.1 \times 10^{3} / \mu 1\right)$. Es posible que el estrés durante la captura y las condiciones climáticas influenciaran los valores de heterófilos (Cabrera et al., 2011). Asimismo, los linfocitos están influenciados por la edad y el aumento se observa en inflamaciones, parasitosis, infecciones virales, neoplasias, cicatrización y estivación (Martínez et al., 2013).

Los valores de proteínas totales fueron ligeramente mayores que los indicados por Rossini et al. (2011); sin embargo, esta variación no sería indicativa de alguna patología. No obstante, hipoalbuminemia ha sido descrita en casos de deshidratación y vitelogénesis (Pereira y de Oliveria, 2007).

\section{Agradecimientos}

Los autores manifiestan su agradecimiento al personal del Centro de Rescate Taricaya y al Sr. Jorge Anco por su valiosa colaboración durante el desarrollo de la investigación. 


\section{Literatura Citada}

1. Arcila VH, Navarro R, Real W, Rincón M, Villamizar M. 2005. Hematología y química sérica en hembras quelonios (Trachemys scripta callirostris) en la ribera del río Lebrija, Puerto Wilches (Santander). Spei Domus 1: 35-43. doi: 10.16925/issn.17947928

2. Cabrera M, Li O, Gálvez, H, Sánchez $N$, Rojas G. 2011. Valores hematológicos de la tortuga motelo (Geochelone denticulata) mantenida en cautiverio. Rev Inv Vet Peru 22: 144150. doi: 10.15381/rivep.v22i2.287

3. Copete-Sierra M. 2013. Aspectos generales de la evaluación hematológica en fauna silvestre y no convencional. Mem Conf Interna Med Aprovech Fauna Silv Exót Conv 9: 17-55.

4. Escobedo A, Gonzáles M. 2008. Estado poblacional del caimán, Caiman crocodilus, en el Refugio Nacional de Vida Silvestre Caño Negro, Costa Rica. Rev Meso Conserv 1(1): 13-20.

5. [ISIS] International Species Information System. 2004. Reference ranges for physiological data values of Caiman crocodilus, USA. [Internet]. Disponible en: http://www2.isis.org/ s u p port/medarks/Page s / Reference\%20Ranges.aspx

6. [IUCN] International Union for Conservation of Nature. 1986. Crocodiles their ecology, management, and conservation. Switzerland: International Union for Conservation of Nature and Natural Resources. [Internet]. Disponible en: https://portals.iucn.org/library/ sites/library/files/documents/NS-1989001.pdf

7. Martínez A, Lavín S, Cuenca R. 2013. La bioquímica sanguínea en clínica de reptiles. Consulta Difus Vet 200:31-40.

8. Nagy K, Medica P. 1986. Physiological ecology of desert tortoises in southern Nevada. Herpetologica 42: 73-92.

9. Pereira N, De Oliveira A. 2007. Patologia clínica. En: Silivino Z, Ramos J, Catao-Dias J (eds). Tratado de animais selvagens. Medicina Veterinária. Vol II. Sao Paulo, Brazil: Roca.

10. Rossini M, García G, Rojas J, Zerpa H. 2011. Hematologic and serum biochemical reference values for the wild spectacled caiman, Caiman crocodilus crocodilus, from the Venezuelan plains. Vet Clin Pathol 40: 374-379. doi: 10.1111/ j.1939-165X.2011.00344.X

11. Rueda-Almonacid J, Carr J, Mittermeier R, Mast R, Vogt R. 2007. Las tortugas y los cocodrilianos de los países andinos de trópico. Bogotá, Colombia: Conservation International. $537 \mathrm{p}$.

12. Sallés J. 2013. Aspectos generales de la evaluación hematológica en fauna silvestre y no convencional. Mem Conferencia Interna en Medicina y Aprovechamiento de Fauna Silvestre, Exótica y no Convencional 9(1): 17-55.

13. Velasco A, Ayarzagüena J. 1995. Situación actual de las poblaciones de baba (Caiman crocodilus) sometidas a aprovechamiento comercial en los Llanos Venezolanos. Cali: Asociación Amigos de Doñana. $71 \mathrm{p}$. 